\title{
ABELIAN GROUPS WHOSE SUBGROUP LATTICE IS THE UNION OF TWO INTERVALS
}

\author{
SIMION BREAZ and GRIGORE CĂLUGĂREANU
}

(Received 15 February 2003; revised 21 July 2003)

Communicated by E. A. O'Brien

\begin{abstract}
In this note we characterize the abelian groups $G$ which have two different proper subgroups $N$ and $M$ such that the subgroup lattice $L(G)=[0, M] \cup[N, G]$ is the union of these intervals.

2000 Mathematics subject classification: primary 06C99, 20K10, 20K27.

Keywords and phrases: subgroup lattice, interval, torsion abelian group, cocyclic groups.

For every subgroup $H$ of an arbitrary group $G$, the interval $[H, G]$ is a compactly generated (algebraic) sublattice in the subgroup lattice $L(G)$.

After 1989, when Tuma [4] showed that every algebraic lattice is isomorphic to an interval in the subgroup lattice of some group (improving Whitman's theorem [5]every lattice is isomorphic to a sublattice of the subgroup lattice of a group-as far as possible), an increasing role of intervals, in subgroup lattices of groups, was noticed.

In [1], an arbitrary group $G$ was called a $B P$-group if it has a proper subgroup $H$ such that the subgroup lattice $L(G)$ is the union of the intervals $[1, H]$ and $[H, G]$ (that is, any subgroup of $G$ is either contained in $H$ or contains $H$ ). The subgroup $H$ was called a breaking point for the lattice $L(G)$. It was pointed out that the abelian BP-groups are the nonsimple cocyclic groups (that is, up to isomorphism, $\mathbb{Z}\left(p^{k}\right)$ with $k>1$ or $\infty$ ).

Roland Schmidt suggested the study of finite groups which satisfy a weaker condition: groups $G$ having two proper subgroups $N$ and $M$ such that every subgroup $H$ of $G$ either contains $N$ or is contained in $M$. In this situation the subgroup lattice $L(G)$ is again union of two intervals, namely $[1, M]$ and $[N, G]$ (such groups appeared in the study of affinities of groups—see for example [3, 9.4.14]—but there are much more examples of this kind).
\end{abstract}

(C) 2005 Australian Mathematical Society 1446-7887/05 $\$$ A2.00+0.00 
In this paper, instead of finite groups, we characterize the abelian groups which share this property. Our result is the following:

THEOREM 1. An abelian group $G$ has two proper subgroups $N \neq M$ such that the subgroup lattice $L(G)=[0, M] \cup[N, G]$ if and only if $G$ is a torsion group with a primary component $G_{p} \cong \mathbb{Z}\left(p^{n}\right) \oplus B, n \in \mathbb{N}^{*} \cup\{\infty\}$ such that $p^{l} B=0$ holds for a nonnegative integer $l<n$.

Additive notation is used and from now on, 'group' means 'abelian group', $\mathbb{N}$ denotes the set of all nonnegative integers, $\mathbb{P}$ denotes the set of all prime numbers and standard interval notation is used. We denote by $h_{p}(b)$ the $p$-height of $b$.

We first mention the following simple necessary condition: $N$ must be cyclic. Indeed, take $x \in G \backslash M$. Then $\langle x\rangle \in[0, M]$ being not possible, $\langle x\rangle \in[N, G]$ or $N \leq\langle x\rangle$.

Next, notice there are three distinct possibilities with respect to subgroups $N$ and $M$
(A) $N$ and $M$ are not comparable;
(B) $M<N$;
(C) $N<M$ (for example, the above mentioned example [3]).

\section{Abelian groups with (A)}

In this section we suppose $M$ and $N$ are not comparable and $L(G)=[0, M] \cup$ $[N, G]$. In this case $[0, M] \cap[N, G]=\emptyset$ (otherwise $N \leq M$ ). We list a few straightforward remarks:

(a) $M \cap N$ is the largest element in $[0, N)$ and $M+N$ is the smallest element in $(M, G]$.

(b) $L(M+N)=[0, M] \cup[N, N+M]$, that is, $N+M$ has property (A).

(c) $L(G /(M \cap N))=[0, M /(M \cap N)] \cup[N /(M \cap N), G /(M \cap N)]$, that is, $G /(M \cap N)$ has property (A).

(d) $(M+N) /(M \cap N)$ has property (A).

Actually, more can be proved:

LEMMA 1.1. If $L(G)=[0, M] \cup[N, G]$, there is a prime number $p$ such that

(a) $N$ is a (co)cyclic $p$-group and $M \cap N=p N$ is maximal in $N$;

(b) $G / M$ and $G /(M+N)$ are $p$-groups.

ProOf. (a) We have already noticed that $N$ has to be cyclic. By the above remark (a), $N$ is a (co)cyclic $p$-group (for a suitable prime number $p$ ). Moreover, since $M \cap N$ is its largest (proper) subgroup, $p N=M \cap N$. 
To prove (b), we observe that $G / M$ is a cocyclic group since it has a smallest subgroup, namely $(M+N) / M$. Moreover, since $(M+N) / M \cong N /(N \cap M) \cong \mathbb{Z}(p)$, $G / M$ is a cocyclic $p$-group, and so $G /(M+N)$ has the same property.

Therefore the subgroup lattice is represented by the diagram shown in Figure 1.

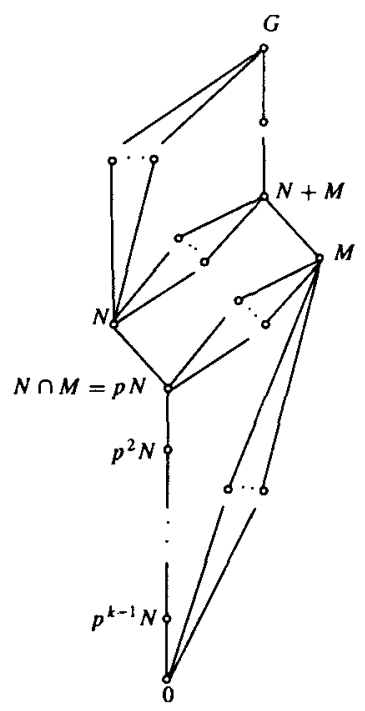

Figure 1.

If $N \simeq \mathbb{Z}\left(p^{k}\right)$ it is readily seen that for $k=1, N$ is minimal and hence the sum $N+M$ is direct (otherwise $N \cap M=N$ and $N, M$ are comparable). Actually this is the only case $N \cap M=0$.

The following lemma will be used in the proofs of the main results of both this and next sections.

LEMMA 1.2. For a group $G$ and $g \in G$, let $p$ be a prime such that $K=G /\langle g\rangle$ is cocyclic $p$-group. If $h_{p}(g) \neq 0$ and $G$ is not infinite cyclic, then $G=H_{1} \oplus H_{2}$ for cocyclic $p$-group $H_{1}$ and finite cyclic group $H_{2}$ of coprime order with $p$ such that $H_{2} \leq\langle g\rangle\left(H_{2}=0\right.$ is not excluded $)$.

PROOF. Since for cocyclic group $G$ the decomposition is trivial, suppose $G$ is not cocyclic (and so $g \neq 0$ ). As $r(G) \leq r(K)+r(\langle g\rangle)=2$, we have $r(G)=2$ and by $r_{0}(G)=r_{0}(\langle g\rangle)+r_{0}(K) \leq 1$, we obtain $G=H_{1} \oplus H_{2}$ with $r\left(H_{1}\right)=r\left(H_{2}\right)=1$, that is, each $H_{i}$ is cocyclic or infinite cyclic (if $r_{0}(G)=1$, the torsion subgroup of $G$ is cocyclic, hence $G$ splits). If $g=h_{1}+h_{2}$ with $h_{i} \in H_{i}$, since $h_{p}(g) \geq 1$, there exist $x_{1} \in H_{1}$ and $x_{2} \in H_{2}$ such that $p x_{1}=h_{1}$ and $p x_{2}=h_{2}$. Moreover, $L(G /\langle g\rangle)$ is a chain and we can suppose $x_{2}+\langle g\rangle \in\left(\left\langle x_{1}\right\rangle+\langle g\rangle\right) /\langle g\rangle$. 
Thus $x_{2} \in\left\langle x_{1}\right\rangle+\langle g\rangle$ and $x_{2}=s x_{1}+t g$ or $p x_{2}=s p x_{1}+t p g$ for suitable integers $s, t$. Hence $h_{2}=s h_{1}+t p\left(h_{1}+h_{2}\right)$ and, the sum $H_{1} \oplus H_{2}$ being direct, $(t p-1) h_{2}=0$.

If $h_{2}=0$ then $g \in H_{1}$ and $K$ is cocyclic if and only if $\langle g\rangle=H_{1}$ or $H_{2}=0$. In the first case $h_{p}(g)=0$, hence $H_{2}=0$ and $G=H_{1}$ is a cocyclic $p$-group (since, by hypothesis, $G$ is not infinite cyclic).

If $h_{2} \neq 0$, the order of $h_{2}$ (say $l$ ) is finite and coprime with $p$. Therefore, $H_{2}$ is a cocyclic $q$-group (if $l$ is a power of the prime $q$ ) and this implies $H_{2} \leq\langle g\rangle$ (otherwise $G /\langle g\rangle$ is not $p$-group). Hence there exists a nonzero integer $k$ such that $h_{2}=k h_{1}+k h_{2}$, and so $k h_{1}=0$. Then $H_{1}$ is also cocyclic and necessarily a $p$-group.

Here is the structure theorem for case (A):

THEOREM 1.1. A group $G$ satisfies (A) if and only if $G$ is torsion with a cocyclic primary component and $r(G)>1$.

ProOF. According to Lemma 1.1, let $p$ be a prime such that $N=\langle a\rangle$ is cyclic of order $p^{k}$. If $m \in M \backslash N$ then $m+a \notin M$ (since $a \notin M$ ) and $N \leq\langle m+a\rangle$. Since $N \neq 0$ is torsion, $m+a$ and therefore $m$ are of finite order. Hence $M$ and, together with $G / M, G$ are torsion.

Further, we show that $M_{p} \subseteq N$. Indeed, if $m \in M_{p}$, again, $N \subseteq\langle a+m\rangle$ so that $a=s(a+m)$ and $(1-s) a=s m \in N \cap M=p N$ for a suitable nonzero integer $s$. Thus $s \equiv 1(\bmod p)$ and let $t$ be an inverse of $s$ modulo the order of $m \in M_{p}$. Thus $m=t s m=t(1-s) a \in N$.

Now, $N$ and $M$ being not comparable, $M_{p} \subset N$ and hence

$$
p N=M \cap N=M_{p} \cap N=M_{p}
$$

Since $M_{p}=p N \leq G_{p}$, Lemma 1.2 shows that $G_{p}$ is a cocyclic group.

Conversely, suppose $G=G_{p} \oplus K$ with $G_{p} \simeq \mathbb{Z}\left(p^{l}\right), K \neq 0, K_{p}=0$ and take $N=G_{p}[p]=\langle a\rangle$ and $M=K$. If $H$ is a subgroup of $G$ such that $H \not K$ we show $N \leq H$.

Indeed, since $H \not K$, there is an element $h \in H \backslash K$. If this element decomposes as $h=g_{p}+k\left(g_{p} \in G_{p}, k \in K\right)$, then $g_{p} \neq 0$ and for a suitable multiple $p^{s} h=p^{s}\left(g_{p}+k\right)$ we have $0 \neq p^{s} g_{p} \in N$ respectively $p^{s} k \in K$. Since $K$ is torsion and $K_{p}=0$, denoting by $u$ the order of $p^{s} k, u$ and $p$ are coprime and $u p^{s} g_{p} \in H$. Finally, $p^{s} g_{p} \in H$ and thus $N=\left\langle p^{s} g_{p}\right\rangle \leq H$.

REMARKS. (1) The referee pointed out that a proof in Case (A) can be reduced to the proof of Case (B) using Lemma 1.1. Our proof uses Lemma 1.2 in both cases.

(2) With above notations, $G / M=\bigoplus_{q \in \mathbb{P}}\left(G_{q} / M_{q}\right)$ is a $p$-group. Hence $G_{q}=M_{q}$ for all primes $q \neq p$ and $M=p N \oplus\left(\bigoplus_{q \neq p . q \in \mathbb{P}} G_{q}\right)$. 


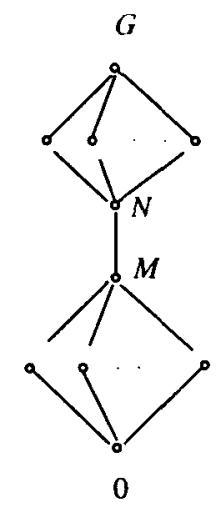

FIGURE 2.

\section{Abelian groups with (B)}

Now we deal with subgroup lattices of the type shown in Figure 2. Here again $[0, M] \cap[N, G]=\emptyset$.

Although the following result was already stated in [1], we supply a specific 'abelian' proof.

LEMMA 2.1. $G$ is an abelian BP-group if and only if there is a prime $p$ and $k \in \mathbb{N}^{*} \cup\{\infty\}, k \geq 2$ such that $G \simeq \mathbb{Z}\left(p^{k}\right)$.

Proof. If $L(G)=[0, H] \cup[H, G]$, then (as noticed in the introduction) $H$ is a cyclic subgroup. If $p$ is a prime such that $p H \neq H$, then $H / p H$ is simple, and using again $L(G)=[0, H] \cup[H, G]$, it is the smallest nonzero subgroup of $G / p H$. Hence $G / p H$ is cocyclic and, having elements of order $p$ (in $H / p H$ ), must be a $p$ group. Since an infinite cyclic group is not a BP-group, using Lemma 1.2, we obtain $G=H_{1} \oplus H_{2}$ with cocyclic $p$-group $H_{1}$, cyclic $q$-group $H_{2}, q$ and $p$ are coprime and $H_{2} \leq p H \leq H$. Obviously, $H_{1} \not L H$ (otherwise $G=H$ ) so that $H_{2} \leq H \leq H_{1}$. This implies $H_{2}=0$, and so $G$ is cocyclic. Since $\mathbb{Z}(p)$ is not satisfying (B), $G$ has the requested form.

The converse is immediate (the subgroup lattice of $\mathbb{Z}\left(p^{n}\right)$ with $n \in \mathbb{N} \cup\{\infty\}, n \geq 2$ is a chain with at least 3 elements).

Using this we obtain at once

THEOREM 2.1. A group satisfies (B) if and only if $G \simeq \mathbb{Z}\left(p^{n}\right)$ with $n \geq 3$.

Proof. If $L(G)=[0, M] \cup[N, G]$ and $M \leq N$ then $L(G)=[0, N] \cup[N, G]$ 


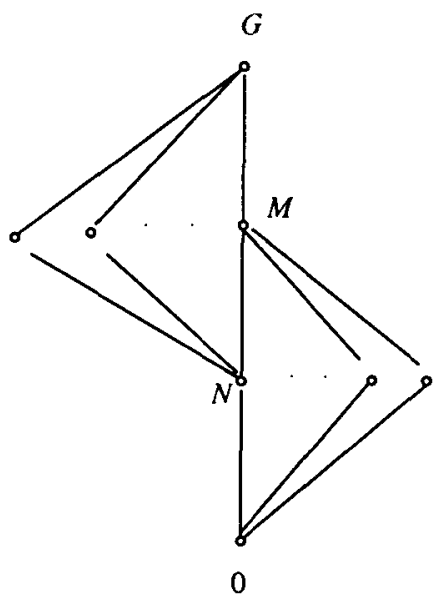

FIGURE 3.

and so $G$ is a BP-group. Hence $G$ is cocyclic. Since the conditions $0 \neq M \neq N \neq G$ require at least 4 elements in $L(G), G \simeq \mathbb{Z}\left(p^{n}\right)$ with $n \geq 3$.

The converse is obvious.

\section{Abelian groups with (C)}

In this section we consider two proper subgroups $N<M$ such that $L(G)=$ $[0, M] \cup[N, G]$. Thus the subgroup lattice looks as shown in Figure 3 .

Now $L(G)=[0, M] \cup[N, G]$ and $[0, M] \cap[N, G]=[M, N]$. Moreover, $[0, N] \subseteq$ $[0, M]$ and $[M, G] \subseteq[N, G]$.

THEOREM 3.1. If a group $G$ satisfies $(C)$ then $G$ is a torsion group and there exists a prime p such that $G_{p}$ is a BP-group or satisfies $(\mathrm{C})$. Conversely, if $G$ is a torsion group, $G_{p} \neq G$ for a prime $p$ and $G_{p}$ is a BP-group or satisfies $(C)$, then $G$ satisfies $(C)$.

Proof. Let $0<N<M<G$ be such that $L(G)=[0, M] \cup[N, G]$.

If $G$ is not a torsion group, there exists an infinite order element $x \in G$ such that $x \notin M$ (otherwise, since the infinite order elements generate any group, $M=G$ ). Then $0<N \leq M \cap\langle x\rangle<\langle x\rangle$. If $L \leq\langle x\rangle$ then $L \leq M$ or $N \leq L$, hence $L \leq M \cap\langle x\rangle$ or $N \leq L$. Therefore $\langle x\rangle$ is a BP-group or satisfies (C), but it is easy to see that no infinite cyclic group satisfies these properties (as for (C), if $0<n \mathbb{Z}<m \mathbb{Z}<\mathbb{Z}$ and $p$ is a prime not dividing $n$, then $p \mathbb{Z} \notin[0, m \mathbb{Z}] \cup[n \mathbb{Z}, \mathbb{Z}])$. This contradiction shows that $G$ is a torsion group. 
Suppose no component $G_{p}$ is a BP-group or satisfies (C). Since $M \neq G$, there exists a prime $p$ such that $M_{p} \neq G_{p}$. If $N_{p}=0$, then $G_{p} \subseteq M\left(N \subseteq G_{p}\right.$ is not possible, $N$ being a proper subgroup), hence $M_{p}=G_{p}$. Therefore $0<N_{p} \leq M_{p}<G_{p}$ and $L\left(G_{p}\right) \neq\left[0, M_{p}\right] \cup\left[N_{p}, G_{p}\right]$. Then we can find $H_{p} \leq G_{p}$ such that $H_{p} \backslash M_{p} \neq \emptyset$ and $N_{p} \backslash H_{p} \neq \emptyset$. It follows $H_{p} \backslash M \neq \emptyset$ and $N \backslash H_{p} \neq \emptyset$, a contradiction.

Conversely, suppose $G$ is torsion and $G_{p}$ is a BP-group or satisfies (C). Then we can find subgroups $0<N_{p} \leq M_{p}<G_{p}$ such that $L\left(G_{p}\right)=\left[0, M_{p}\right] \cup\left[N_{p}, G_{p}\right]$. Set $M=M_{p} \oplus\left(\bigoplus_{q \neq p} G_{q}\right)$ and $N=N_{p}$. Thus $0<N<M<G$. If $H \leq G$, then $H=H_{p} \oplus\left(\bigoplus_{q \neq p} H_{q}\right)$ with $H_{p} \leq G_{p}$ and $\bigoplus_{q \neq p} H_{q} \leq \bigoplus_{q \neq p} G_{q}$. If $N_{p} \leq H_{p}$, then $H \in[N, G]$ and if $H_{p} \leq M_{p}$, then $H \leq M_{p} \oplus\left(\bigoplus_{q \neq p} H_{q}\right) \leq M_{p} \oplus\left(\bigoplus_{q \neq p} G_{q}\right)=M$. Actually, $G_{p} \neq G$ is needed only for a BP-group $G_{p}$ not satisfying (C).

THEOREM 3.2. A p-group $G$ satisfies (C) if and only if $G \cong \mathbb{Z}\left(p^{n}\right) \oplus B$ such that (i) $B \neq 0, n \in \mathbb{N}^{*} \cup\{\infty\}$ and $p^{l} B=0$ holds for a positive integer $l<n$, or (ii) $B=0$ and $n>2$.

ProOF. If $G$ satisfies (C), we can suppose $N=\langle a\rangle \cong \mathbb{Z}(p)$. Let $l>0$ be the smallest positive integer such that there exists $x \in G \backslash M$ with $p^{l} x=a$. Let $b \in G[p] \backslash\langle a\rangle$ and suppose $h_{p}(b) \geq l$. Then $b=p^{l} y$ for some $y \in M$ (if $y \notin M$ we have $a \in\langle y\rangle$, hence the rank of $\langle y\rangle[p]$ is at least 2 , a contradiction). Thus $x+y \notin M$, and there exists a positive integer $k$ such that $k x+k y=a$. If $k=p^{r} m$ with $\operatorname{gcd}(m ; p)=1$ then $p^{r}(m x+m y)=a$, hence $l \leq r$. Moreover, $l \leq r$ implies $k y \in\langle a\rangle$ and $a \in\langle y\rangle$ follows, a contradiction. Then $h_{p}(b)<l$ for all $b \in G[p] \backslash\langle a\rangle$ and so $p^{l} G[p]=\langle a\rangle$. Hence $p^{l} G$ is a cocyclic group.

If $p^{l} G$ is a cyclic group then $G$ is bounded and (using [2, 27.2]) $G=H \oplus B$ where $H \cong \mathbb{Z}\left(p^{n}\right)$ with $n \geq l+1, a \in H$ and $p^{l} B=0$ (otherwise there is $b \in B[p]$ with $\left.h_{p}(b) \geq l\right)$. If $p^{l} G$ is a quasicyclic group, then $G=p^{l} G \oplus B$ and $p^{l} B=0$.

Moreover, if $B=0$ then $G \cong \mathbb{Z}\left(p^{n}\right)$ and condition $M \neq N$ implies $n>2$.

Conversely, if $B=0$ then $G$ satisfies condition (C) for $N=p^{n-1} G$ and $M=p G$. If $B \neq 0$ we choose $G=H \oplus B$ with $H \simeq \mathbb{Z}\left(p^{n}\right), 0<l<n$ such that $p^{l} B=0$, $N=H[p]=\langle a\rangle \cong \mathbb{Z}(p)$ and $M=A+B$ where $A$ is the subgroup of $H$ of order $p^{l}$ (obviously containing $N$ - the subgroup lattice of $H$ being a chain with a smallest element). If $X$ is a subgroup of $G$ such that $X \notin[0, M]$, then there exists $x=h+b \in X \backslash M$ with $h \in H$ and $b \in B$ such that $p^{r}=\operatorname{ord}(h)>p^{l}$ (otherwise $h \in A$ and $x \in M)$. By $p^{l} B=0$ hypothesis, $0 \neq p^{r-1} x=p^{r-1} h \in H[p]=N$, hence $\left\langle p^{r-1} h\right\rangle=N$ is included in $X$.

The only BP-groups which do not satisfy (B), nor $(C)$ are $\mathbb{Z}\left(p^{2}\right)$ for any prime number $p$. Hence 


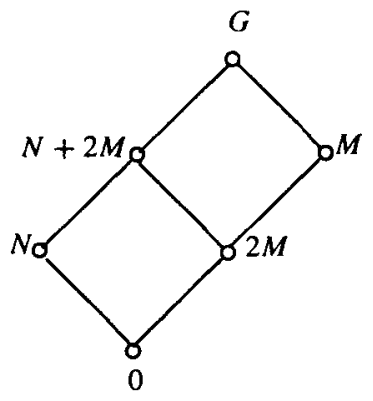

(a)

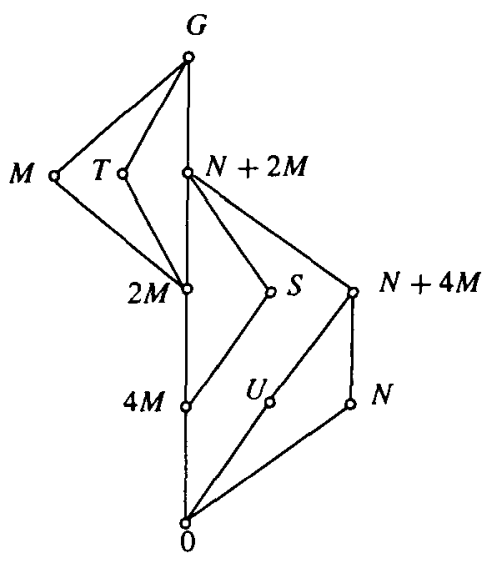

(b)

Figure 4.

COROLlaRY 3.1. A group $G$ satisfies (C) if and only if it is a torsion group with a primary component $\mathbb{Z}\left(p^{n}\right)$ for $n \geq 3$, or $G_{p} \cong \mathbb{Z}\left(p^{n}\right) \oplus B$ with $n>1$ or $\infty$ and $p^{l} B=0$ holds for a nonnegative integer $l<n$.

\section{Comments}

1. There are groups satisfying both conditions (A) and (C). As an example take

$$
G=\mathbb{Z}(12)=\langle a, b \mid 3 a=4 b=0\rangle .
$$

Denoting by $N=\langle a\rangle$ and $M=\langle b\rangle$ the subgroup lattice looks as shown in Figure 4 (a). Thus $L(G)=[0, M] \cup[N, G]$ for $(\mathrm{A})$, and $L(G)=[0, N+2 M] \cup[2 M, G]$ for (C).

2. If a group $G$ satisfies, say, the condition (C) the pair $M, N$ of subgroups is not necessarily unique. As an example, take the group

$$
G=\mathbb{Z}(2) \oplus \mathbb{Z}(8)=\langle a, b \mid 2 a=8 b=0\rangle .
$$

If we denote by $N=\langle a\rangle, M=\langle b\rangle, S=\langle a+2 b\rangle, T=\langle a+b\rangle, U=\langle a+4 b\rangle$, the subgroup lattice is now as shown in Figure 4 (b) and

$$
L(G)=[0, N+2 M] \cup[2 M, G]=[0, N+2 M] \cup[4 M, G] .
$$

3. Our results generalize to lattices with 0 and 1 , more or less arbitrary. In what follows we state some of these lattice versions.

- If a lattice $L$ satisfies condition (A), that is, $L=[0, m] \cup[n, 1]$ with incomparable elements $m, n$ then 


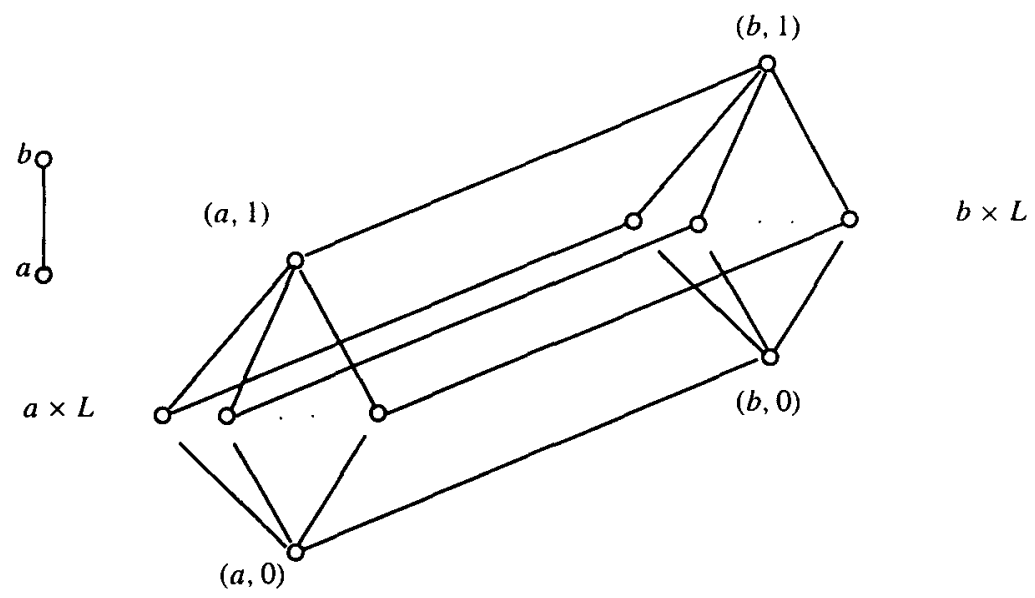

FIGURE 5.

(a) $[0, m \vee n]=[0, m] \cup[n, m \vee n]$ that is, $[0, m \vee n]$ satisfies condition (A);

(b) $[m \wedge n, 1]=[m \wedge n, m] \cup[n, 1]$ that is, $[m \wedge n, 1]$ satisfies condition (A);

(c) $[m \wedge n, m \vee n]$ satisfies condition (A).

- Every direct product of two lattices, the first being a finite chain and the second having 0 and 1 , satisfies condition $(A)$.

Proof. One uses the diagram shown in Figure 5 (for the sake of simplicity we have considered a chain with only two elements).

Denoting the chain by $\{a, b\}$ and using elements in the Cartesian product $\{a, b\} \times L$, decomposition in the required intervals is $[(a, 0),(a, 1)] \cup[(b, 0),(b, 1)]$.

A family of torsion groups is said to be coprime if the orders of elements in any two members are coprime. Using an early Theorem of Suzuki (see [3]): the groups with decomposable subgroup lattices are exactly the direct sums of coprime groups, we have an alternative proof for sufficiency of Theorem 1.1 in the special case $k=1$.

PROOF FOR SUFFICIENCY OF THEOREM $1.1(k=1)$. Let $G$ be a torsion group of rank $r(G)>1$ with a simple $p$-component, that is $G=N \oplus M$ with $|N|=p$ and $M_{p}=0$. Thus $N$ and $M$ are coprime, $L(G) \simeq L(N) \times L(M)$ and $L(N)$ is a chain with two elements. Applying the previous result, $L(G)$ satisfies condition (A).

- Complemented lattices are not satisfying condition (C).

- Let $\left\{L_{i}, i \in I\right\}$ be an arbitrary set of bounded (that is, with $0_{i}$ and $1_{i}$ ) lattices, at least one of these satisfying condition (C). Then the direct product $L=\prod_{i \in I} L_{i}$ satisfies condition (C). Conversely, if $L$ satisfies condition (C), that is, $L=[\mathbf{0}, \alpha] \cup$ $[\beta, 1]$ and for an index $j \in I, 0_{j}<\beta_{j}<\alpha_{j}<1_{j}$, then $L_{j}$ satisfies condition (C). 
- If a lattice satisfies condition (C), that is, $L=[0, m] \cup[n, 1]$, then $m$ is essential and $n$ is superfluous in $L$. Moreover, every element disjoint with $n$ belongs to $[0, m]$.

Finally we mention the lattice version of our initial proof of case (A):

- Let $L$ be a modular lattice, $n$ an atom and $m$ a dual atom in $L$ such that $1=n \vee m$ and $n \wedge m=0$. Then $L=[0, m] \cup[n, 1]$ if and only if for every element $v$ in $[0, m], n$ has a unique (relative) complement (namely $v$ ) in the sublattice $[0, n \vee v]$.

Using this, one can show that, excepting the case $1=n \vee m$ and $n \wedge m=0$, (C) follows from (A).

\section{Acknowledgement}

Thanks are due to the referee for his (her) valuable suggestions and improvements.

\section{References}

[1] G. Călugăreanu and M. Deaconescu, 'Breaking points in subgroup lattices', in: Proceedings of Groups St Andrews 2001 in Oxford (Cambridge University Press, Cambridge, 2003) pp. 59-62.

[2] L. Fuchs, Infinite abelian groups, Vol. 1, 2 (Academic Press, New York, 1970, 1973).

[3] R. Schmidt, Subgroup lattices of groups, Expositions in Mathematics 14 (Walter de Gruyter, 1994).

[4] J. Tuma, 'Intervals in subgroup lattices of infinite groups', J. Algebra (2) 125 (1989), 367-399.

[5] P. M. Whitman, 'Lattices, equivalence relations, and subgroups', Bull. Amer. Math. Soc. 52 (1946), $507-522$.

Faculty of Mathematics and Informatics

Babes-Bolyai University

Cluj-Napoca

Romania

e-mail: bodo@math.ubbcluj.ro
Dept. Mathematics and Computer Sci

Faculty of Science

Kuwait University

State of Kuwait

e-mail: calu@mcs.sci.kuniv.edu.kw 